Pacific

Journal of

Mathematics

ON COMPOSITION OPERATORS WHICH PRESERVE BMO

YASUhIRo Gotoh

Volume $201 \quad$ No. 2

December 2001 


\title{
ON COMPOSITION OPERATORS WHICH PRESERVE BMO
}

\author{
Yasuhiro Gotoh \\ Dedicated to Professor Kôzô Yabuta on his sixtieth birthday
}

\begin{abstract}
We characterize the Lebesgue measurable maps between Euclidean spaces which preserve BMO.
\end{abstract}

\section{Introduction.}

For a subdomain $D$ of $\mathbf{R}^{n}, n \geq 1$, let $\operatorname{BMO}(D)$ be the space of all locally integrable functions $f$ on $D$ satisfying

$$
\|f\|_{*}=\|f\|_{* . D}=\sup _{Q \subset D}|Q|^{-1} \int_{Q}\left|f-f_{Q}\right| d x<\infty,
$$

where $|Q|$ is the $n$-dimensional Lebesgue measure of $Q, f_{Q}=|Q|^{-1} \int_{Q} f d x$, and the supremum is taken over all closed cubes $Q \subset D$ with sides parallel to the coordinate axes.

Let $D$ and $D^{\prime}$ be subdomains of $\mathbf{R}^{m}$ and $\mathbf{R}^{n}, m, n \geq 1$, respectively. We say that a map $F: D \rightarrow D^{\prime}$ is measurable if $F^{-1}(E)$ is measurable for each measurable subset $E$ of $D^{\prime}$. We say that a measurable map $F: D \rightarrow D^{\prime}$ is a $B M O$ map if i) for each null set $E \subset D^{\prime}, F^{-1}(E)$ is also a null set, and furthermore, ii) for each $\operatorname{BMO}\left(D^{\prime}\right)$ function $f, C_{F}(f)=f \circ F$ belongs to $\operatorname{BMO}(D)$. The condition i) guarantees the uniqueness of the function $f \circ F$. From the closed graph theorem each BMO map $F$ induces a bounded operator $C_{F}$ between BMO spaces.

Various partial results are known for the characterization of BMO maps. It seems, however, that we do not know almost anything yet for noncontinuous BMO maps. The main purpose of the present paper is to give a characterization of BMO maps $F: \mathbf{R}^{m} \rightarrow \mathbf{R}^{n}, m, n \geq 1$ (Theorem 3.1).

Our argument depends on the following two celebrated results for BMO; a growth estimation for BMO functions due to John-Nirenberg, and the existence of certain extremal BMO functions due to Uchiyama (Propositions 4.1 and 4.2).

The present paper is organized as follows. First, we give various examples of BMO maps in $\S 2$. The main results of the present paper are given in $\S 3$. The following $\S 4$ is devoted to their proofs. Finally, in $\S 5$ we give a remark on BMO maps which are homeomorphisms between intervals. 
In the following, a cube implies a closed cube with sides parallel to the coordinate axes, $t Q$ denotes the cube with the same center as $Q$ and expanded by a constant factor $t>0$, and we use the letter $C$ to denote a positive constant which may vary from place to place unless stated otherwise, that is, $f \leq 2 C$ implies $f \leq C$, on the other hand, $f \leq 2 C_{2}$ does not necessarily mean $f \leq C_{2}$. Also we sometimes write " $F: D \rightarrow D^{\prime}$ " even if $F(D) \not \subset D^{\prime}$ under the assumption that both $A=F(D) \backslash D^{\prime}$ and $F^{-1}(A)$ are null sets. For instance, we may write $F: \mathbf{R} \rightarrow(0, \infty), F(x)=|x|$, instead of $F: \mathbf{R} \rightarrow[0, \infty), F(x)=|x|$.

The author would like to thank the referee for his helpful comments and suggestions.

\section{Examples.}

In the present section we give various examples of BMO maps.

Example 2.1. a) Let $F: D_{1} \times D_{2} \rightarrow D_{1}, D_{1} \subset \mathbf{R}^{m}, D_{2} \subset \mathbf{R}^{n}$, be the canonical projection. Then $F$ is a $\mathrm{BMO}$ map satisfying $\left\|C_{F}\right\|=1$. In particular, if $D_{2}=\mathbf{R}^{n}$, then $\left\|C_{F}(f)\right\|_{*}=\|f\|_{*}$ holds for each $f \in \operatorname{BMO}\left(D_{1}\right)$.

b) Let $F: D \rightarrow D^{\prime}$ be the inclusion map. Then $F$ is a BMO map satisfying $\left\|C_{F}\right\|=1$.

Example 2.2. Let $F: D \rightarrow D^{\prime}$ be a homeomorphism between subdomains of $\mathbf{R}^{n}, n \geq 2$. If $F$ is quasiconformal, then $F$ is a BMO map satisfying $\left\|C_{F}\right\| \leq C\left(n, K_{F}\right)$, where $K_{F}$ is the maximal dilatation of $F$. Conversely, if $F$ is a BMO map satisfying i) for each null set $E, F^{-1}(E)$ is also a null set, ii) $F$ is ACL, iii) $F$ is differentiable a.e., then $F$ is a quasiconformal map satisfying $K_{F} \leq C\left(n,\left\|C_{F}\right\|\right)$ (Reimann [13]).

Example 2.3. Let $F$ be a homeomorphism of $\mathbf{R}$. Then $F$ is a BMO map if and only if we can take constants $K, \alpha>0$ so that

$$
\frac{\left|F^{-1}(E \cap I)\right|}{\left|F^{-1}(I)\right|} \leq K\left(\frac{|E \cap I|}{|I|}\right)^{\alpha}
$$

holds for each pair of a measurable subset $E$ of $\mathbf{R}$ and an interval $I$ (Jones [11]). Note that (2) holds if and only if $F^{-1}$ is absolutely continuous and its derivative $\left(F^{-1}\right)^{\prime}$ (or $\left.-\left(F^{-1}\right)^{\prime}\right)$ is an $A_{\infty}$ weight (cf. (4)). In this case $F^{-1}$ also satisfies the same condition, and so $F$ induces a bijection of $\operatorname{BMO}(\mathbf{R})$.

Jones gave no explicit relation between the constants $K, \alpha$ above and $\left\|C_{F}\right\|$. In $\S 3$ we show, however, that his argument implicitly gives the following estimations: If (2) holds, then $\left\|C_{F}\right\| \leq C K / \alpha$ for some universal constant $C>0$; conversely, if $F$ is a BMO map, then we can take constants $K, \alpha$ so that $K=C_{1}$ and $\alpha=C_{2} /\left\|C_{F}\right\|$, where $C_{k}>0, k=1,2$, are universal constants. Hence $\left\|C_{F}\right\|$ and $\inf (K / \alpha)$ are comparable with universal 
constant factors, where the infimum is taken over all pairs of $K, \alpha$ satisfying (2) (Theorem 5.3) (cf. Mayer-Zinsmeister [12]).

Fominykh [3] gave a sufficient condition for spherically continuous maps between (finite or infinite) open intervals to be BMO maps, which partially extends Jones' result.

Example 2.4. Let $F: D \rightarrow D^{\prime}$ be a nonconstant holomorphic map between plane domains. Then $F$ is a BMO map if and only if we can take an integer $p>0$ so that for each disk $B$ satisfying $2 B \subset D, F$ is $p$-valent on $B$. In particular, a holomorphic map $F: \mathbf{C} \rightarrow \mathbf{C}$ is a BMO map if and only if it is a polynomial (Gotoh $[8]$ ).

Thus, whether a given nonconstant holomorphic map $F: D \rightarrow D^{\prime}$ between plane domains is a BMO map or not is independent of the choice of its target $D^{\prime}$. The following example shows that this does not extend to hold for general maps.

Example 2.5. a) Let $D=\left\{x \in \mathbf{R}^{2}|1<| x \mid<2\right\}, I=\left\{\left(0, x_{2}\right) \in \mathbf{R}^{2} \mid-2<\right.$ $\left.x_{2}<-1\right\}$, and $D_{0}=D \backslash I$. Let $F$ satisfy $F(x)=x$ on $D_{0}$ and $F(x) \in D_{0}$ on $I$. Then $F: D \rightarrow D$ is a BMO map, and $F: D \rightarrow D_{0}$ is not a BMO map, because $\operatorname{BMO}(D) \neq \operatorname{BMO}\left(D_{0}\right)$.

b) Let $F: D \rightarrow D^{\prime}$ be a BMO map. Let $D_{0}^{\prime}$ be a subdomain of $D^{\prime}$ satisfying $F(D) \subset D_{0}^{\prime}$. Assume that each $\operatorname{BMO}\left(D_{0}^{\prime}\right)$ function is the restriction of some $\operatorname{BMO}\left(D^{\prime}\right)$ function. (Such domains $D_{0}^{\prime}$ are characterized as relative uniform domains with respect to $D^{\prime}$ (Gotoh [7].) For instance, uniform domains $D_{0}^{\prime}$ satisfy this condition (Proposition 3.4). In this case $F: D \rightarrow D^{\prime}$ is a BMO map if and only $F: D \rightarrow D_{0}^{\prime}$ is a BMO map.

Example 2.6. Let $D=\mathbf{R}^{n-1} \times(0, \infty)$ be the upper half space. Then for each $f \in \operatorname{BMO}(D)$, its symmetric extension $g, g(x, y)=f(x, y)$ on $D$ and $g(x, y)=f(x,-y)$ on $\mathbf{R}^{n} \backslash D$, is a $\operatorname{BMO}\left(\mathbf{R}^{n}\right)$ function satisfying $\|f\|_{*, \mathbf{R}^{n}} \leq C\|f\|_{*, D}$, where $C>0$ is a universal constant, which is called a reflection principle for BMO. In other words, the two-sheeted folding $\operatorname{map} F: \mathbf{R}^{n} \rightarrow D, F(x, y)=(x,|y|)$, is a BMO map satisfying $\left\|C_{F}\right\| \leq C$ (cf. Reimann-Rychener [14]).

Example 2.7. Let $D$ be a quasidisk, that is, $D$ is the image of the upper half plane under a quasiconformal map of $\overline{\mathbf{R}^{2}}=\mathbf{R}^{2} \cup\{\infty\}$. Let $\tau: \overline{\mathbf{R}^{2}} \rightarrow \overline{\mathbf{R}^{2}}$ be the quasiconformal reflection with respect to $\partial D$. Then from Examples 2.2 and 2.6 the two-sheeted folding map $F: \mathbf{R}^{2} \rightarrow D, F(x)=x$ on $D$ and $F(x)=\tau(x)$ on $\mathbf{R}^{n} \backslash D$, is a BMO map.

Example 2.8. a) Let $D=\left\{r<|x|<r^{\prime}\right\} \subset \mathbf{R}^{n}, n \geq 2$. Let $a=r^{\prime} / r$ and set $D_{k}=\left\{a^{k} r<|x|<a^{k+1} r\right\}, k \in \mathbf{Z}$. We define an infinite-sheeted folding map $F: \mathbf{R}^{n} \rightarrow D$ as follows: Set $F(x)=x / a^{2 k}$ on $D_{2 k}$ and $F(x)=$ $F\left(\tau_{k}(x)\right)$ on $D_{2 k+1}$, where $\tau_{k}$ is the reflection with respect to the sphere 
$\left\{|x|=a^{2 k+1} r\right\}$. Then $F$ is a BMO map and $\left\|C_{F}\right\| \leq \frac{a C}{a-1}$, where $C=$ $C(n)>0$. This is a consequence of the reflection principle, the removability of one point for BMO, and Proposition 5.2 below.

b) We define an infinite-sheeted folding map $F: \mathbf{R} \rightarrow(0,1)$ as follows: Set $F(x)=x$ on $[0,1], F(x)=2-x$ on $[1,2]$, and $F(x)=F(x-2 k)$, $2 k \leq x \leq 2 k+2, k \in \mathbf{Z}$. Then $F$ is a BMO map. On the other hand, $F \times i d_{\mathbf{R}}: \mathbf{R} \times \mathbf{R} \rightarrow(0,1) \times \mathbf{R}$ is not a BMO map: Let $f\left(x_{1}, x_{2}\right)=x_{2}$. Then $C_{F \times i d_{\mathbf{R}}}(f)\left(x_{1}, x_{2}\right)=x_{2}$. Thus $f \in \operatorname{BMO}((0,1) \times \mathbf{R})$ and $C_{F \times i d_{\mathbf{R}}}(f) \notin$ $\operatorname{BMO}(\mathbf{R} \times \mathbf{R})$.

There are essentially non-continuous BMO maps.

Example 2.9. a) Let $\tau$ be a Möbius transformation of $\overline{\mathbf{R}^{n}}=\mathbf{R}^{n} \cup\{\infty\}$, $n \geq 2$. Let $D$ be an arbitrary subdomain of $\mathbf{R}^{n}$ and $D^{\prime}=\tau(D) \backslash\{\infty\}$. Then $F=\tau \mid D: D \rightarrow D^{\prime}$ is a BMO map. This is a consequence of Example 2.2 and the removability of one point for BMO. (cf. Reimann-Rychener [14]. Also see Lemma 5.1 below.) For instance, $x \mapsto x /|x|^{2}$, which is discontinuous at the origin under the Euclidean topology, induces a bijection between $\operatorname{BMO}(\{|x|<1\})$ and $\operatorname{BMO}(\{|x|>1\})$.

b) Let $F: \mathbf{R} \rightarrow(0,1)$ be the infinite-sheeted folding map in Example 2.8 b). Then $G: \mathbf{R} \rightarrow(0,1), G(x)=F(1 / x)$, is a BMO map which is discontinuous at the origin even under the spherical topology.

Moreover, there are BMO maps between plane domains with essential singularities.

Example 2.10. Let $F(z)=\mathcal{P}(1 / z)$, where $\mathcal{P}$ is the Weierstrass $\mathcal{P}$-function. Then $F: \mathbf{C} \rightarrow \mathbf{C}$ is a BMO map having the origin as an essential singularity. Another example is given by the Blaschke product $F: \mathbf{C} \rightarrow \mathbf{C}$,

$$
F(z)=\prod_{k=0}^{\infty} \frac{z-2^{-k} i}{z+2^{-k} i} \prod_{k=1}^{\infty} \frac{2^{k} i-z}{2^{k} i+z} .
$$

(See the next example.) Moreover, for an arbitrary plane domain $D$ and an arbitrary sequence $\left\{z_{k}\right\} \subset D, z_{k} \rightarrow \partial D$, there exists a BMO meromorphic map $F: D \rightarrow \mathbf{C}$ having $\left\{z_{k}\right\}$ as simple poles satisfying $\left\|C_{F}\right\| \leq C$, where $C>0$ is a universal constant (Gotoh $[6],[8]$ ).

Contrary to the case of holomorphic maps between plane domains (Example 2.4), it seems difficult to estimate the operator norms for rational maps $F: \mathbf{C} \rightarrow \mathbf{C}$. As to this we only know the following.

Example 2.11. Let $F$ be a finite Blaschke product on the unit disk $\Delta$. Let $t_{\zeta}, \zeta \in \Delta$, denote the Carleson constant associated with the zeros of the Blaschke product $(F-\zeta) /(1-\bar{\zeta} F)$. Let $s_{F}=\sup _{\zeta \in \Delta} t_{\zeta}$. Then for the operator norm $\left\|C_{F}\right\|$ of the map $F: \mathbf{C} \rightarrow \mathbf{C}$, we have $\left\|C_{F}\right\| \leq C_{1}\left(s_{F}\right)$ and $s_{F} \leq C_{2}\left(\left\|C_{F}\right\|\right)$. In particular, we can show that there exists a sequence of 
rational maps $F_{k}: \mathbf{C} \rightarrow \mathbf{C}$, $\operatorname{deg} F_{k}=k$, satisfying $\left\|C_{F_{k}}\right\| \leq C$, where $C>0$ is a universal constant (Gotoh $[\mathbf{6}]$ ).

For related topics, see Astala [1], Smith [15], and Mayer-Zinsmeister [12].

\section{Main theorem.}

We say that a domain $D \subset \mathbf{R}^{n}$ is admissible if $D$ is an increasing limit of some sequence of cubes. For instance, $\mathbf{R}^{n}$, half spaces with sides parallel to the coordinate axes, and open cubes are admissible.

Theorem 3.1 (Main Theorem). For a measurable map $F: D \rightarrow D^{\prime}, D \subset$ $\mathbf{R}^{m}, D^{\prime} \subset \mathbf{R}^{n}$, we consider the following conditions:

(a) We can take constants $K, \alpha>0$ so that for an arbitrary pair of measurable subsets $E_{1}, E_{2}$ of $D^{\prime}$ we have

$$
\sup _{Q \subset D} \min _{k=1,2} \frac{\left|F^{-1}\left(E_{k}\right) \cap Q\right|}{|Q|} \leq K\left(\sup _{Q^{\prime} \subset D^{\prime}} \min _{k=1,2} \frac{\left|E_{k} \cap Q^{\prime}\right|}{\left|Q^{\prime}\right|}\right)^{\alpha},
$$

where the suprema are taken over all cubes $Q \subset D$ and $Q^{\prime} \subset D^{\prime}$ respectively;

(b) We can take constants $\gamma, 0<\gamma<1 / 4$, and $\lambda>0$ so that for an arbitrary pair of measurable subsets $E_{1}, E_{2}$ of $D^{\prime}$ satisfying

$$
\sup _{Q^{\prime} \subset D^{\prime}} \min _{k=1,2} \frac{\left|E_{k} \cap Q^{\prime}\right|}{\left|Q^{\prime}\right|}<\lambda,
$$

we have

$$
\sup _{Q \subset D} \min _{k=1,2} \frac{\left|F^{-1}\left(E_{k}\right) \cap Q\right|}{|Q|}<\gamma
$$

where the suprema are taken over all cubes $Q \subset D$ and $Q^{\prime} \subset D^{\prime}$ respectively;

(c) $F$ is a BMO map.

Then we have $(\mathrm{a}) \Rightarrow(\mathrm{b}) \Rightarrow(\mathrm{c})$. Moreover, if we can take an admissible domain $D_{0}^{\prime}$ satisfying $F(D) \subset D_{0}^{\prime} \subset D^{\prime}$, then all these conditions are equivalent.

In particular, all the conditions above are equivalent if $D^{\prime}$ is admissible. The implication (a) $\Rightarrow$ (b) is trivial. We show that (a) implies (c) with $\left\|C_{F}\right\| \leq C(m, n) K / \alpha$ (Lemma 4.8). Furthermore, if we can take an admissible domain $D_{0}^{\prime}$ satisfying $F(D) \subset D_{0}^{\prime} \subset D^{\prime}$, then we show that (c) implies (a) with constants $K=K(m, n)$ and $\alpha=C(m, n) /\left\|C_{F}\right\|$ (Lemma 4.10). Thus we have:

Corollary 3.2. If we can take an admissible domain $D_{0}^{\prime}$ satisfying $F(D) \subset$ $D_{0}^{\prime} \subset D^{\prime}$, then the operator norm $\left\|C_{F}\right\|$ and $\inf (K / \alpha)$ are comparable with 
constant factors depending only on $m$ and $n$, where the infimum is taken over all pairs of constants $K, \alpha$ satisfying the estimation (3).

Let $F$ be a homeomorphism of $\mathbf{R}$. Then the condition (2) in Example 2.3 implies (3) with the same constants $K, \alpha$. And so we may regard the Main Theorem as an extension of Jones' result.

We say that a weight $w$ is an $A_{\infty}$ weight on $D$ if we can take a constant $\alpha, K>0$ so that

$$
\frac{\int_{E \cap Q} w d x}{\int_{Q} w d x} \leq K\left(\frac{|E \cap Q|}{|Q|}\right)^{\alpha}
$$

holds for each pair of a measurable set $E \subset D$ and a cube $Q \subset D$. A weight $w$ is an $A_{\infty}$ weight if and only if we can take constants $\varepsilon, \delta, 0<$ $\varepsilon, \delta<1$, so that for each pair of $E, Q$ satisfying $|E \cap Q| /|Q|<\delta$, we have $\int_{E \cap Q} w d x / \int_{Q} w d x<\varepsilon$. The equivalence of the conditions (a) and (b) of the Main Theorem implies the corresponding result holds for BMO maps.

Recall that for a weight $w$ on $D, f=\log w$ belongs to $\operatorname{BMO}(D)$ if and only if $w^{\gamma}$ is an $A_{\infty}$ weight on $D$ for some $\gamma>0$. Hence,

Corollary 3.3. We can add the condition

(d) For each $A_{\infty}$ weight $w$ on $D^{\prime}, w^{\gamma} \circ F$ is an $A_{\infty}$ weight on $D$ for some $\gamma>0$.

to the list of the Main Theorem in the sense that $(\mathrm{c}) \Leftrightarrow(\mathrm{d})$ holds.

We say that a domain $D \subset \mathbf{R}^{n}$ is uniform if

$$
k_{D}(x, y) \leq C \log \left(\frac{d(x, \partial D)+d(y, \partial D)+|x-y|}{\min \{d(x, \partial D), d(y, \partial D)\}}\right), \quad x, y \in D,
$$

holds for some $C>0$, where $k_{D}$ is the quasihyperbolic metric on $D$. Uniform domains are invariant under quasiconformal maps on $\overline{\mathbf{R}^{n}}$. Half spaces are uniform domains. In the case of a simply connected plane domain $D, D$ is uniform if and only if $D$ is a quasidisk. The uniformness can be characterized by the BMO extension property.

Proposition 3.4 (Jones [10]). A domain $D \subset \mathbf{R}^{n}$ is uniform if and only if each $\mathrm{BMO}(D)$ function is the restriction of some $\mathrm{BMO}\left(\mathbf{R}^{n}\right)$ function.

In this case, for each $g \in \operatorname{BMO}(D)$ we can take $f \in \operatorname{BMO}\left(\mathbf{R}^{n}\right), f \mid D=g$, so that $\|f\|_{*} \leq C\|g\|_{*}$, where $C>0$ is a constant depending only on $n$ and the constant of uniformness.

Corollary 3.5. Let $D$ and $D^{\prime}$ be subdomains of $\mathbf{R}^{m}$ and $\mathbf{R}^{n}$ respectively. Assume that $D^{\prime}$ is uniform. Then for a measurable map $F: D \rightarrow D^{\prime}$, the following conditions are equivalent: 
(a) We can take constants $K, \alpha>0$ so that for an arbitrary pair of measurable subsets $E_{1}, E_{2}$ of $D^{\prime}$ we have

$$
\sup _{Q \subset D} \min _{k=1,2} \frac{\left|F^{-1}\left(E_{k}\right) \cap Q\right|}{|Q|} \leq K\left(\sup _{Q^{\prime} \subset \mathbf{R}^{n}} \min _{k=1,2} \frac{\left|E_{k} \cap Q^{\prime}\right|}{\left|Q^{\prime}\right|}\right)^{\alpha},
$$

where the suprema are taken over all cubes $Q \subset D$ and $Q^{\prime} \subset \mathbf{R}^{n}$ respectively;

(b) We can take constants $\gamma, 0<\gamma<1 / 4$, and $\lambda>0$ so that for an arbitrary pair of measurable subsets $E_{1}, E_{2}$ of $D^{\prime}$ satisfying

$$
\sup _{Q^{\prime} \subset \mathbf{R}^{n}} \min _{k=1,2} \frac{\left|E_{k} \cap Q^{\prime}\right|}{\left|Q^{\prime}\right|}<\lambda,
$$

we have

$$
\sup _{Q \subset D} \min _{k=1,2} \frac{\left|F^{-1}\left(E_{k}\right) \cap Q\right|}{|Q|}<\gamma
$$

where the suprema are taken over all cubes $Q \subset D$ and $Q^{\prime} \subset \mathbf{R}^{n}$ respectively;

(c) $F$ is a BMO map;

(d) $G=i \circ F: D \rightarrow \mathbf{R}^{n}$ is a BMO map, where $i: D \rightarrow \mathbf{R}^{n}$ is the inclusion map.

We cannot replace the condition " $Q^{\prime} \subset \mathbf{R}^{n \text { " }}$ in (a) (and in (b)) above with " $Q{ }^{\prime} \subset D^{\prime \prime}$ " (Example 4.11).

Remark 3.6. We may replace the assertion (a) of the Main Theorem with

$\left(\mathrm{a}^{\prime}\right)$ For each $N \geq 2$ we can take constants $K, \alpha>0$ so that for arbitrary measurable subsets $E_{1}, \ldots, E_{N}$ of $D^{\prime}$ we have

$$
\sup _{Q \subset D} \min _{1 \leq k \leq N} \frac{\left|F^{-1}\left(E_{k}\right) \cap Q\right|}{|Q|} \leq K\left(\sup _{Q^{\prime} \subset D^{\prime}} \min _{1 \leq k \leq N} \frac{\left|E_{k} \cap Q^{\prime}\right|}{\left|Q^{\prime}\right|}\right)^{\alpha},
$$

where the suprema are taken over all cubes $Q \subset D$ and $Q^{\prime} \subset D^{\prime}$ respectively,

or

$\left(\mathrm{a}^{\prime \prime}\right)$ The assertion $\left(\mathrm{a}^{\prime}\right)$ holds for some $N \geq 2$.

Similarly, we may replace the assertion (b) of the Main Theorem with

(b') For each $N \geq 2$ we can take constants $\gamma, 0<\gamma<1 / 4$, and $\lambda>0$ so that for arbitrary measurable subsets $E_{1}, \ldots, E_{N}$ of $D^{\prime}$ satisfying

$$
\sup _{Q^{\prime} \subset D^{\prime}} \min _{1 \leq k \leq N} \frac{\left|E_{k} \cap Q^{\prime}\right|}{\left|Q^{\prime}\right|}<\lambda
$$


we have

$$
\sup _{Q \subset D} \min _{1 \leq k \leq N} \frac{\left|F^{-1}\left(E_{k}\right) \cap Q\right|}{|Q|}<\gamma
$$

where the suprema are taken over all cubes $Q \subset D$ and $Q^{\prime} \subset D^{\prime}$ respectively,

or

$\left(\mathrm{b}^{\prime \prime}\right)$ The assertion $\left(\mathrm{b}^{\prime}\right)$ holds for some $N \geq 2$.

The implications $\left(\mathrm{a}^{\prime}\right) \Rightarrow(\mathrm{a}) \Rightarrow\left(\mathrm{a}^{\prime \prime}\right),\left(\mathrm{b}^{\prime}\right) \Rightarrow(\mathrm{b}) \Rightarrow\left(\mathrm{b}^{\prime \prime}\right),(\mathrm{a}) \Rightarrow(\mathrm{b}),\left(\mathrm{a}^{\prime}\right)$ $\Rightarrow\left(\mathrm{b}^{\prime}\right)$, and $\left(\mathrm{a}^{\prime \prime}\right) \Rightarrow\left(\mathrm{b}^{\prime \prime}\right)$ are trivial. Furthermore, we obtain $\left(\mathrm{a}^{\prime \prime}\right) \Rightarrow(\mathrm{a})$ and $\left(\mathrm{b}^{\prime \prime}\right) \Rightarrow(\mathrm{b})$ by setting $E_{2}=E_{3}=\cdots=E_{N}$. In the next section we show (b) $\Rightarrow$ (c), and (c) $\Rightarrow$ (a) (under the additive assumption). It is easy to check that we can show $(\mathrm{c}) \Rightarrow\left(\mathrm{a}^{\prime}\right)$ in the same way.

Note that we can also rewrite Corollaries 3.2 and 3.5 similarly.

\section{Proofs of the Main Theorem and Corollary 3.5.}

The following two results play fundamental roles in the proof of the Main Theorem. The latter one shows that the growth estimation of BMO functions given by the former one is remarkably precise.

Proposition 4.1 (John-Nirenberg [9]). Let $f \in \operatorname{BMO}(D), D \subset \mathbf{R}^{n}$, and $Q \subset D$ be a cube. Then

$$
\left|\left\{x \in Q|| f(x)-f_{Q} \mid \geq t\right\}\right| \leq C_{1}|Q| \exp \left(-C_{2} \frac{t}{\|f\|_{*}}\right), \quad t \geq 0,
$$

where $C_{1}, C_{2}>0$ are constants depending only on $n$.

Proposition 4.2 (Uchiyama [17], cf. Garnett-Jones [4]). Let D be an admissible subdomain of $\mathbf{R}^{n}$. Let $N \geq 2, t>1$, and $E_{1}, \ldots, E_{N}$ be measurable subsets of D satisfying

$$
\sup _{Q \subset D} \min _{1 \leq k \leq N} \frac{\left|E_{k} \cap Q\right|}{|Q|} \leq 2^{-n t},
$$

where the supremum is taken over all cubes $Q \subset D$. Then there exist $\operatorname{BMO}(D)$ functions $f_{1}, \ldots, f_{N}$ satisfying $\sum_{k=1}^{N} f_{k}=1$ and

$$
0 \leq f_{k} \leq 1, \quad f_{k}=0 \quad \text { on } E_{k}, \quad\left\|f_{k}\right\|_{*} \leq C / t, \quad(0 \leq k \leq N),
$$

where $C=C(n, N)>0$.

Garnett-Jones showed the assertion when $D=\mathbf{R}^{n}, N=2$ and $E_{1} \subset Q$, $E_{2}=\mathbf{R}^{n} \backslash 2 Q$. Uchiyama extended their result to the form above.

First, we give a variant of the John-Nirenberg Theorem. 
Lemma 4.3. Let $f \in \operatorname{BMO}(D), D \subset \mathbf{R}^{n}$, and $Q \subset D$ be a cube. Then

$$
\begin{aligned}
& \min \{|\{x \in Q \mid f(x) \geq t\}|,|\{x \in Q \mid f(x) \leq s\}|\} \\
& \leq C_{1}|Q| \exp \left(-C_{2} \frac{t-s}{\|f\|_{*}}\right), \quad-\infty<s \leq t<\infty,
\end{aligned}
$$

where $C_{1}, C_{2}>0$ are constants depending only on $n$.

Proof. We may assume $f_{Q} \leq(s+t) / 2$. Then from the John-Nirenberg theorem we have

$$
\begin{aligned}
|\{x \in Q \mid f(x) \geq t\}| & \leq\left|\left\{x \in Q|| f(x)-f_{Q} \mid \geq \frac{t-s}{2}\right\}\right| \\
& \leq C|Q| \exp \left(-C \frac{t-s}{\|f\|_{*}}\right) .
\end{aligned}
$$

Conversely,

Lemma 4.4. Let $f, f(x) \neq \pm \infty$ (a.e.), be a measurable function on a domain $D \subset \mathbf{R}^{n}$. Assume that there exist constants $C_{1}, C_{2}>0$ such that for each cube $Q \subset D$ we have

$$
\begin{aligned}
& \min \{|\{x \in Q \mid f(x) \geq t\}|,|\{x \in Q \mid f(x) \leq s\}|\} \\
& \leq C_{1}|Q| e^{-C_{2}(t-s)}, \quad-\infty<s \leq t<\infty .
\end{aligned}
$$

Then $f$ is a $\operatorname{BMO}(D)$ function satisfying $\|f\|_{*} \leq 4\left(C_{1}+1\right) C_{2}^{-1} \exp \left(2 C_{2}\right)$.

This is a direct consequence of the following.

Lemma 4.5. Let $\lambda: \mathbf{R} \rightarrow[0,1]$ be a nonconstant, non-decreasing function. Assume that there exist constants $C_{1}, C_{2}>0$ such that

$$
\min (\lambda(s), 1-\lambda(t)) \leq C_{1} e^{-C_{2}(t-s)}, \quad-\infty<s \leq t<\infty .
$$

Then we can take $t_{0} \in \mathbf{R}$ so that

$$
\max \left(\lambda\left(t_{0}-t\right), 1-\lambda\left(t_{0}+t\right)\right) \leq\left(C_{1}+1\right) e^{2 C_{2}} e^{-C_{2} t}, \quad t \geq 0 .
$$

Proof. Since $\lambda$ is nonconstant, $\lambda(t) \rightarrow 0(t \rightarrow-\infty)$, and $\lambda(t) \rightarrow 1(t \rightarrow \infty)$. Let $s_{k}=\sup \{t \mid \lambda(t) \leq 1-\lambda(t+k)\}, k \geq 1$. Then $s_{k}$ is non-increasing, $s_{k}+k$ is non-decreasing, and

$$
\lambda\left(s_{k}-1\right) \leq 1-\lambda\left(s_{k}+k-1\right), \quad \lambda\left(s_{k}+1\right)>1-\lambda\left(s_{k}+k+1\right) .
$$

Set $t_{0}=s_{1}$. First, assume $k \leq t<k+1, k \geq 2$. Then

$$
\begin{gathered}
1-\lambda\left(t_{0}+t\right) \leq 1-\lambda\left(s_{k-1}+k\right) \leq C_{1} e^{-C_{2}(k-1)} \leq C_{1} e^{2 C_{2}} e^{-C_{2} t}, \\
\lambda\left(t_{0}-t\right) \leq \lambda\left(s_{k-1}-1\right) \leq C_{1} e^{-C_{2}(k-1)} \leq C_{1} e^{2 C_{2}} e^{-C_{2} t} .
\end{gathered}
$$

Next, if $0 \leq t<2$, then $\max \left\{\lambda\left(t_{0}-t\right), 1-\lambda\left(t_{0}+t\right)\right\} \leq 1 \leq e^{2 C_{2}} e^{-C_{2} t}$. 
Proof of Lemma 4.4. Set $\lambda(t)=|\{x \in Q \mid f(x) \leq t\}| /|Q|$. Then $\lambda$ satisfies the assumption of Lemma 4.5 with the same constants $C_{1}, C_{2}$. Thus we can take $t_{0}$ so that

$$
\max \left\{\lambda\left(t_{0}-t\right), 1-\lambda\left(t_{0}+t\right)\right\} \leq\left(C_{1}+1\right) e^{2 C_{2}} e^{-C_{2} t}, \quad t \geq 0 .
$$

And so

$$
\mu(t):=\left|\left\{x \in Q|| f(x)-t_{0} \mid \geq t\right\}\right| \leq 2\left(C_{1}+1\right)|Q| e^{2 C_{2}} e^{-C_{2} t}, \quad t \geq 0 .
$$

Hence

$$
\int_{Q}\left|f-f_{Q}\right| d x \leq 2 \int_{Q}\left|f-t_{0}\right| d x=2 \int_{0}^{\infty} \mu(t) d t \leq 4\left(C_{1}+1\right) C_{2}^{-1} e^{2 C_{2}}|Q| .
$$

Lemma 4.6. Let $F: D \rightarrow D^{\prime}$ satisfy the condition (a) of the Main Theorem. Then $K \geq 1$ and $\alpha \leq 1$.

Proof. We obtain $K \geq 1$ by setting $E_{1}=E_{2}=D^{\prime}$.

Next, assume $\alpha>1$. Let $Q_{0}=[p, q] \times P_{0} \subset \mathbf{R} \times \mathbf{R}^{n-1}=\mathbf{R}^{n}$ be a cube in $D^{\prime}$. Let $l=q-p$. Let $I_{1}=[p, p+l / 4], I_{2}=[q-l / 4, q]$. We decompose $I$ into $2^{s}$ subintervals $J_{k}=\left[p+2^{-s}(k-1) l, p+2^{-s} k l\right], 1 \leq k \leq 2^{s}$, where $s$ is a sufficiently large integer. Let $E_{k}=\left(J_{k} \times \mathbf{R}^{n-1}\right) \cap D^{\prime}$. Let $Q$ be a cube in $D$. Let $k_{0}$ be the integer $k$ which maximizes $\left|F^{-1}\left(E_{k}\right) \cap Q\right|, 1 \leq k \leq 2^{s}$. Then

$$
\sup _{Q^{\prime} \subset D^{\prime}} \min \left\{\frac{\left|E_{k} \cap Q^{\prime}\right|}{\left|Q^{\prime}\right|}, \frac{\left|E_{k_{0}} \cap Q^{\prime}\right|}{\left|Q^{\prime}\right|}\right\} \leq \frac{4}{2^{s}}
$$

holds for each $k \in \Sigma_{1}$ or for each $k \in \Sigma_{2}$, where $\Sigma_{1}=\left\{k \mid J_{k} \subset I_{1}\right\}$ and $\Sigma_{2}=\left\{k \mid J_{k} \subset I_{2}\right\}$. Thus from the assumption we have

$$
\frac{\left|F^{-1}\left(E_{k}\right) \cap Q\right|}{|Q|} \leq K\left(\frac{4}{2^{s}}\right)^{\alpha},
$$

for each $k \in \Sigma_{1}$ or for each $k \in \Sigma_{2}$. It follows from $\sharp \Sigma_{1}=\sharp \Sigma_{2}=2^{s-2}$ that

$$
\min _{j=1,2}\left|F^{-1}\left(\left(I_{j} \times \mathbf{R}^{n-1}\right) \cap D^{\prime}\right) \cap Q\right| \leq 2^{s-2} K\left(\frac{4}{2^{s}}\right)^{\alpha}|Q| \rightarrow 0, \quad s \rightarrow \infty .
$$

Therefore, the measure $\mu(S)=\left|F^{-1}\left(\left(S \times \mathbf{R}^{n-1}\right) \cap D^{\prime}\right) \cap Q\right|$ on $[p, q]$ is absolutely continuous and satisfies $\mu([a, a+t])=0$ or $\mu([a+3 t, a+4 t])=$ 0 for each $a, t$ with $p \leq a<a+4 t \leq q$. Thus $\mu([p, q])=0$, and so $\left|F^{-1}\left(Q_{0}\right) \cap Q\right|=0$. Since $Q$ and $Q_{0}$ are arbitrary, we have $\left|F^{-1}\left(D^{\prime}\right)\right|=0$, which is a contradiction.

Lemma 4.7. Let $F: D \rightarrow D^{\prime}, D \subset \mathbf{R}^{m}, D^{\prime} \subset \mathbf{R}^{n}$, be a BMO map. Then $\left\|C_{F}\right\| \geq 1$. 
Proof. Note that if $E$ is a measurable subset of $D$ satisfying $|E|>0$, $|D \backslash E|>0$, then the characteristic function $f$ of $E$ satisfies $\|f\|_{*}=\|f\|_{*, D}=$ $1 / 2$.

Let $F$ be a BMO map. Fix a cube $Q \subset D$ and set $\lambda(E)=\left|F^{-1}(E) \cap Q\right|$. Then $\lambda$ is an absolutely continuous finite measure on $D^{\prime}$, thus we can take $t_{0} \in \mathbf{R}$ so that $\lambda\left(E_{0}\right)=\lambda\left(D^{\prime} \backslash E_{0}\right)=|Q| / 2$, where $E_{0}=\left(\left(-\infty, t_{0}\right] \times\right.$ $\left.\mathbf{R}^{n-1}\right) \cap D^{\prime}$. Let $f$ be the characteristic function of $E_{0}$. Then $f \circ F$ is the characteristic function of $F^{-1}\left(E_{0}\right)$, and so from the first paragraph we have $\|f\|_{*}=\|f \circ F\|_{*}=1 / 2$, which implies the assertion.

The following lemma shows (a) $\Rightarrow$ (c) of the Main Theorem with an estimation of the operator norm.

Lemma 4.8. Let $F: D \rightarrow D^{\prime}, D \subset \mathbf{R}^{m}, D^{\prime} \subset \mathbf{R}^{n}$, be a measurable map. Assume that there exist constants $K, \alpha>0$ such that for each pair of measurable subsets $E_{1}, E_{2}$ of $D^{\prime}$, we have

$$
\sup _{Q \subset D} \min _{k=1,2} \frac{\left|F^{-1}\left(E_{k}\right) \cap Q\right|}{|Q|} \leq K\left(\sup _{Q^{\prime} \subset D^{\prime}} \min _{k=1,2} \frac{\left|E_{k} \cap Q^{\prime}\right|}{\left|Q^{\prime}\right|}\right)^{\alpha} .
$$

Then $F$ is a BMO map satisfying $\left\|C_{F}\right\| \leq C K / \alpha$, where $C=C(m, n)>0$.

Proof. Assume that $F$ satisfies the assumption of the lemma. Then the inverse image of a null set is trivially a null set.

Let $f \in \operatorname{BMO}\left(D^{\prime}\right), E_{1}=\left\{x \in D^{\prime} \mid f(x) \leq s\right\}, E_{2}=\left\{x \in D^{\prime} \mid f(x) \geq t\right\}$, $-\infty<s \leq t<\infty$, and $Q^{\prime} \subset D^{\prime}$. From Lemma 4.3 we have

$$
\min \left\{\left|E_{1} \cap Q^{\prime}\right|,\left|E_{2} \cap Q^{\prime}\right|\right\} \leq C\left|Q^{\prime}\right| \exp \left(-\frac{C(t-s)}{\|f\|_{*}}\right) .
$$

It follows from the assumption and the fact $\alpha \leq 1$ (Lemma 4.6) that for an arbitrary cube $Q \subset D$ we have

$$
\min \left\{\left|F^{-1}\left(E_{1}\right) \cap Q\right|,\left|F^{-1}\left(E_{2}\right) \cap Q\right|\right\} \leq C K|Q| \exp \left(-\frac{C \alpha(t-s)}{\|f\|_{*}}\right) .
$$

Now, $F^{-1}\left(E_{1}\right)=\{x \in D \mid f(F(x)) \leq s\}, F^{-1}\left(E_{2}\right)=\{x \in D \mid f(F(x)) \geq t\}$, and so from Lemma 4.4 and the fact $K \geq 1$ (Lemma 4.6) we have $F \circ f \in$ $\operatorname{BMO}(D)$ and

$$
\|f \circ F\|_{*} \leq \frac{C K}{\alpha}\|f\|_{*} \exp \left(\frac{C \alpha}{\|f\|_{*}}\right) .
$$

Finally, applying this estimation to $t f, t>0$, and letting $t \rightarrow \infty$, we obtain $\|f \circ F\|_{*} \leq \frac{C K}{\alpha}\|f\|_{*}$.

Next, to show (b) $\Rightarrow$ (c) of the Main Theorem, we need the following. 
Proposition 4.9 (Strömberg $[\mathbf{1 6}]$ ). Let $f$ be a measurable function on $D \subset$ $\mathbf{R}^{n}$. Assume that we can take constants $\gamma, 0<\gamma<1 / 2$, and $\lambda>0$ so that for each cube $Q \subset D$ we have

$$
\inf _{c \in \mathbf{R}}|\{x \in Q|| f(x)-c \mid \geq \lambda\}| \leq \gamma|Q| .
$$

Then $f$ is a $\operatorname{BMO}(D)$ function satisfying $\|f\|_{*} \leq C \lambda$, where $C=C(n, \gamma)>$ 0 .

Proof of Main Theorem (b) $\Rightarrow$ (c). Assume that $F$ satisfies the condition (b) of the Main Theorem. Then the inverse image of a null set is trivially a null set.

Let $f \in \operatorname{BMO}\left(D^{\prime}\right)$. We may assume $\|f\|_{*}=1$. Let $-\infty<s<t<\infty$, $E_{1}=\left\{x \in D^{\prime} \mid f(x) \leq s\right\}, E_{2}=\left\{x \in D^{\prime} \mid f(x) \geq t\right\}$, and $g=C_{F}(f)$. Then from Lemma 4.3 there exists $C_{1}>0$ such that if $t-s \geq C_{1}$, then

$$
\sup _{Q^{\prime} \subset D^{\prime}} \min _{k=1,2} \frac{\left|E_{k} \cap Q^{\prime}\right|}{\left|Q^{\prime}\right|} \leq C e^{-C(t-s)}<\lambda,
$$

and so

$$
\sup _{Q \subset D} \min _{k=1,2} \frac{\left|F^{-1}\left(E_{k}\right) \cap Q\right|}{|Q|}<\gamma
$$

For $Q \subset D$ we set

$$
s_{Q}=\sup \left\{s \in \mathbf{R}||\{x \in Q \mid g(x) \leq s\}|\leq|\left\{x \in Q \mid g(x) \geq s+C_{1}\right\} \mid\right\} .
$$

Since $g \neq \pm \infty$ (a.e.), we have $s_{Q} \neq \pm \infty$. Thus

$$
\left|\left\{x \in Q \mid g(x) \leq s_{Q}-1\right\}\right|<\gamma, \quad\left|\left\{x \in Q \mid g(x) \geq s_{Q}+C_{1}+1\right\}\right|<\gamma,
$$

and so if we set $c_{Q}=s_{Q}+C_{1} / 2$ and $\delta=1+C_{1} / 2$, then

$$
\left|\left\{x \in Q|| g(x)-c_{Q} \mid>\delta\right\}\right| \leq 2 \gamma \quad(<1 / 2) .
$$

Hence $g \in \operatorname{BMO}(D)$ by Proposition 4.9 .

Finally, we show the remaining implication $(c) \Rightarrow(a)$ of the Main Theorem.

Lemma 4.10. Let $F: D \rightarrow D^{\prime}, D \subset \mathbf{R}^{m}, D^{\prime} \subset \mathbf{R}^{n}$, be a BMO map. Assume that there exists an admissible domain $D_{0}^{\prime}$ satisfying $F(D) \subset D_{0}^{\prime} \subset$ $D^{\prime}$. Then there exist constants $K, \beta>0$ depending only on $m$ and $n$ such that for an arbitrary pair of measurable subsets $E_{1}, E_{2}$ of $D^{\prime}$ we have

$$
\sup _{Q \subset D} \min _{k=1,2} \frac{\left|F^{-1}\left(E_{k}\right) \cap Q\right|}{|Q|} \leq K\left(\sup _{Q^{\prime} \subset D^{\prime}} \min _{k=1,2} \frac{\left|E_{k} \cap Q^{\prime}\right|}{\left|Q^{\prime}\right|}\right)^{\beta /\left\|C_{F}\right\|} .
$$


Proof. Let $G: D \rightarrow D_{0}^{\prime}, G(x)=F(x)$. Let $g \in \operatorname{BMO}\left(D_{0}^{\prime}\right)$. Since admissible domains are uniform domains with uniformly bounded constants of uniformness, from Proposition 3.4 we can take $f \in \operatorname{BMO}\left(D^{\prime}\right)$ so that $f \mid D_{0}^{\prime}=g$ and $\|f\|_{*} \leq C\|g\|_{*}$. Thus $\|g \circ G\|_{*}=\|f \circ F\|_{*} \leq C\|f\|_{*}$. Therefore, $G$ is a BMO map satisfying $\left\|C_{G}\right\| \leq C\left\|C_{F}\right\|$.

Let $E_{1}, E_{2}$ be an arbitrary pair of measurable subsets of $D^{\prime}$. Let $E_{k}^{\prime}=$ $E_{k} \cap D_{0}^{\prime}$. Then

$$
2^{-n t}:=\sup _{Q^{\prime} \subset D_{0}^{\prime}} \min _{k=1,2} \frac{\left|E_{k}^{\prime} \cap Q^{\prime}\right|}{\left|Q^{\prime}\right|} \leq \sup _{Q^{\prime} \subset D^{\prime}} \min _{k=1,2} \frac{\left|E_{k} \cap Q^{\prime}\right|}{\left|Q^{\prime}\right|} .
$$

Because of Lemma 4.7, we may assume $t>1$. From the Uchiyama theorem there exist $\operatorname{BMO}\left(D_{0}^{\prime}\right)$ functions $f_{1}, f_{2}$ satisfying $f_{1}+f_{2}=1$ and

$$
0 \leq f_{k} \leq 1, \quad f_{k}=0 \text { on } E_{k}^{\prime}, \quad\left\|f_{k}\right\|_{*} \leq C / t, \quad(k=1,2) .
$$

Let $g_{k}=f_{k} \circ G$. Then $g_{1}+g_{2}=1$ and

$$
0 \leq g_{k} \leq 1, \quad g_{k}=0 \quad \text { on } G^{-1}\left(E_{k}^{\prime}\right), \quad\left\|g_{k}\right\|_{*} \leq C\left\|C_{G}\right\| / t, \quad(k=1,2) .
$$

Let $Q$ be an arbitrary cube in $D$. Since $\left(g_{1}\right)_{Q}+\left(g_{2}\right)_{Q}=1$, we may assume $\left(g_{1}\right)_{Q} \geq 1 / 2$. Then from the John-Nirenberg theorem we have

$$
\begin{aligned}
\left|G^{-1}\left(E_{1}^{\prime}\right) \cap Q\right| & \leq\left|\left\{x \in Q|| g_{1}(x)-\left(g_{1}\right)_{Q} \mid \geq 1 / 2\right\}\right| \\
& \leq C|Q| \exp \left(-\frac{C}{\left\|g_{1}\right\|_{*}}\right) \leq C|Q| \exp \left(-\frac{C t}{\left\|C_{G}\right\|}\right),
\end{aligned}
$$

and so we obtain

$$
\begin{aligned}
\sup _{Q \subset D} \min _{k=1,2} \frac{\left|F^{-1}\left(E_{k}\right) \cap Q\right|}{|Q|} & =\sup _{Q \subset D} \min _{k=1,2} \frac{\left|G^{-1}\left(E_{k}^{\prime}\right) \cap Q\right|}{|Q|} \\
& \leq C\left(\sup _{Q^{\prime} \subset D_{0}^{\prime}} \min _{k=1,2} \frac{\left|E_{k}^{\prime} \cap Q^{\prime}\right|}{\left|Q^{\prime}\right|}\right)^{\frac{C}{\left\|C_{G}\right\|}} \\
& \leq C\left(\sup _{Q^{\prime} \subset D^{\prime}} \min _{k=1,2} \frac{\left|E_{k} \cap Q^{\prime}\right|}{\left|Q^{\prime}\right|}\right)^{\frac{C}{\left\|C_{F}\right\|}} .
\end{aligned}
$$

Proof of Corollary 3.5. (a) $\Rightarrow$ (b) is trivial. (c) $\Leftrightarrow$ (d) follows from Proposition 3.4. (d) $\Rightarrow$ (a) is a consequence of the Main Theorem.

Finally, assume that (b) holds. Let $E_{1}$ and $E_{2}$ be measurable subsets of $\mathbf{R}^{n}$ satisfying

$$
\sup _{Q^{\prime} \subset \mathbf{R}^{n}} \min _{k=1,2} \frac{\left|E_{k} \cap Q^{\prime}\right|}{\left|Q^{\prime}\right|}<\lambda .
$$


Then $E_{k}^{\prime}=E_{k} \cap D^{\prime}$ satisfies the assumption. Thus

$$
\sup _{Q \subset D} \min _{k=1,2} \frac{\left|G^{-1}\left(E_{k}\right) \cap Q\right|}{|Q|}=\sup _{Q \subset D} \min _{k=1,2} \frac{\left|F^{-1}\left(E_{k}^{\prime}\right) \cap Q\right|}{|Q|}<\gamma,
$$

and so $G$ satisfies the condition (b) of the Main Theorem. Hence (d) follows.

Example 4.11. Let $D^{\prime}=\left\{x \in \mathbf{R}^{2}|| x_{1}|<4,| x_{2} \mid<5\right\} \backslash\left\{x \in \mathbf{R}^{2} \mid x_{1} \geq\right.$ $\left.0,\left|x_{2}\right| \leq 1\right\}$, where $x=\left(x_{1}, x_{2}\right)$. Then $D^{\prime}$ is a uniform domain. Let $a_{1}=(2,3), a_{2}=(2,-3), E_{1}=\left\{\left|x-a_{1}\right| \leq 1\right\}$, and $E_{2}=\left\{\left|x-a_{2}\right| \leq 1\right\}$. Let $D=\left\{x \in \mathbf{R}^{2}|| x_{1}|<1,| x_{2} \mid<1\right\}$. Let $F: D \rightarrow D^{\prime}$ be a conformal map. Then $F$ is a BMO map. On the other hand, $\min \left\{\left|E_{1} \cap Q^{\prime}\right|,\left|E_{2} \cap Q^{\prime}\right|\right\}=0$ holds for each cube $Q^{\prime} \subset D^{\prime}$. Thus we cannot replace the condition " $Q$ ' $\subset$ $\mathbf{R}^{n "}$ in Corollary 3.5 with $Q^{\prime} \subset D^{\prime}$.

\section{Homeomorphisms between intervals.}

The Main Theorem gives a characterization of BMO maps between (finite or infinite) open intervals. Jones result (Example 2.3) implies that in the case of homeomorphisms of $\mathbf{R}$ we can reduce the condition to a much simpler form. The purpose of the present section is to show that his argument really characterizes BMO maps which are homeomorphisms between general open intervals with an explicit estimation on operator norms.

Recall that the space $\mathrm{BMO}(\mathbf{R})$ is invariant under Möbius transformations of $\overline{\mathbf{R}}=\mathbf{R} \cup\{\infty\}$ (cf. Riemann-Rychener [14]). More generally:

Lemma 5.1. Let $I_{1}$ and $I_{2}$ be open intervals on $\mathbf{R}$. Let $\tau$ be a Möbius transformation of $\overline{\mathbf{R}}$ satisfying $\tau\left(I_{1}\right)=I_{2}$. Then $F=\tau \mid I_{1}: I_{1} \rightarrow I_{2}$ is a BMO map satisfying $C^{-1} \leq\left\|C_{F}(f)\right\|_{*} /\|f\|_{*} \leq C, f \in \operatorname{BMO}\left(I_{2}\right)$, where $C>0$ is a universal constant.

Proof. Let $f \in \operatorname{BMO}\left(I_{2}\right)$. Let $I$ be an interval satisfying $2 I \subset I_{1}$. Then $\max _{I}\left|F^{\prime}\right| \leq C \min _{I}\left|F^{\prime}\right|$, thus

$$
|I|^{-1} \int_{I}\left|f \circ F-f_{F(I)}\right| d x \leq C|F(I)|^{-1} \int_{F(I)}\left|f-f_{F(I)}\right| d x \leq C\|f\|_{*},
$$

and so $\left\|C_{F}(f)\right\| \leq C\|f\|_{*}$ from the proposition below.

Proposition 5.2 (cf. Reimann-Rychener [14]). Let $f \in L_{\mathrm{loc}}^{1}(D), D \subset \mathbf{R}^{n}$, and $t \geq 1$. Assume that

$$
\sup |Q|^{-1} \int_{Q}\left|f-f_{Q}\right| d x \leq \lambda
$$

holds for each $Q$ satisfying $t Q \subset D$. Then $f \in \operatorname{BMO}(D)$ and $\|f\|_{*} \leq C t \lambda$, where $C=C(n)>0$. 
By virtue of the proposition above, repeating the argument of Jones, we can easily extend his result as follows.

Theorem 5.3 (cf. Jones [11]). Let $F: I_{1} \rightarrow I_{2}$ be a homeomorphism between open intervals.

(a) If $I_{1}=\mathbf{R}$ and $I_{2} \neq \mathbf{R}$, then $F$ is not a BMO map.

(b) If $I_{1} \neq \mathbf{R}$ or $I_{2}=\mathbf{R}$, then the following conditions are equivalent:

(i) $F$ is a BMO map;

(ii) There exist constant $K_{0}, \alpha_{0}>0$ such that for each measurable subset $E$ of $I_{2}$ and each subinterval $I$ of $I_{2}$ satisfying $2 F^{-1}(I) \subset I_{1}$, we have

$$
\frac{\left|F^{-1}(E \cap I)\right|}{\left|F^{-1}(I)\right|} \leq K_{0}\left(\frac{|E \cap I|}{|I|}\right)^{\alpha_{0}}
$$

Moreover, if (7) holds, then $\left\|C_{F}\right\| \leq C K_{0} / \alpha_{0}$ for some universal constant $C>0$, and conversely, if $F$ is a $\mathrm{BMO}$ map, then we can take constants $K_{0}, \alpha_{0}$ so that $K_{0}=C_{1}$ and $\alpha_{0}=C_{2} /\left\|C_{F}\right\|$, where $C_{k}>0$, $k=1,2$, are universal constants.

In particular, $\left\|C_{F}\right\|$ and $\inf \left(K_{0} / \alpha_{0}\right)$ are comparable with universal constant factors, where the infimum is taken over all pairs of $K_{0}, \alpha_{0}$ satisfying (7).

Lemma 5.4. Let $I_{0}$ be an interval. Let $J_{1}$ and $J_{2}$ be mutually disjoint subintervals of $I_{0}$. Then

$$
\sup _{I \subset I_{0}} \min _{k=1,2} \frac{\left|J_{k} \cap I\right|}{|I|}=\frac{s}{d\left(J_{1}, J_{2}\right)+2 s},
$$

where $s=\min _{k=1,2}\left|J_{k}\right|$.

Proof of Theorem 5.3. First, assume $I_{1}=\mathbf{R}$ and $I_{2} \neq \mathbf{R}$. From the Möbius invariance of $\mathrm{BMO}$ we may assume $I_{2}=(0, \infty)$ and $F$ is sense preserving. Let $f(x)=\log x$. Then $f \in \operatorname{BMO}\left(I_{2}\right)$. On the other hand, since $g=f \circ F$ is an increasing function satisfying $\lim _{x \rightarrow \infty} g(x)=\infty, \lim _{x \rightarrow-\infty} g(x)=-\infty$, if we set $J_{k}=[-k, k]$ and $E_{k}=[-k,-k / 2] \cup[k / 2, k]$, then

$$
\left|J_{k}\right|^{-1} \int_{J_{k}}\left|g-g_{J_{k}}\right| d x \geq(2 k)^{-1} \int_{E_{k}} \geq 4^{-1}(g(k / 2)-g(-k / 2)) \rightarrow \infty,
$$

as $k \rightarrow \infty$. Thus $F$ is not a BMO map.

Next, assume that $F$ satisfies the condition (ii) of (b). Then $K_{0} \geq 1$ and $\alpha_{0} \leq 1$. Let $I$ be a subinterval of $I_{2}$ satisfying $2 F^{-1}(I) \subset I_{1}$. Let $f \in$ $\operatorname{BMO}\left(I_{2}\right), I^{\prime}=F^{-1}(I), g=f \circ F$, and $E_{t}=\left\{x \in I|| f(x)-f_{I} \mid \geq t\right\}, t \geq 0$. Then from the John-Nirenberg theorem we have $\left|E_{t}\right| \leq C|I| \exp \left(-C t /\|f\|_{*}\right)$, 
thus

$$
\begin{aligned}
\mu(t):=\left|\left\{x \in I^{\prime}|| g(x)-f_{I} \mid \geq t\right\}\right| & =\left|F^{-1}\left(E_{t}\right)\right| \\
& \leq C K_{0}\left|I^{\prime}\right| \exp \left(-\frac{C \alpha_{0} t}{\|f\|_{*}}\right)
\end{aligned}
$$

and so

$$
\left|I^{\prime}\right|^{-1} \int_{I^{\prime}}\left|g-f_{I}\right| d x=\int_{0}^{\infty} \mu(t) d t \leq \frac{C K_{0}}{\alpha_{0}}\|f\|_{*} .
$$

Hence, from Proposition $5.2\|g\|_{*} \leq C K_{0} \alpha_{0}^{-1}\|f\|_{*}$.

Finally, assume that $F$ satisfies the condition (i) of (b). We may assume that $F$ is sense preserving. Let $I_{k}=\left(p_{k}, q_{k}\right), k=1,2$. Let $E$ be a measurable subset of $I_{2}$. Let $I=[a, b]$ be an interval satisfying $2 F^{-1}(I) \subset I_{1}$. Let $l=|I|$ and $l^{\prime}=\left|F^{-1}(I)\right|$. Let $E_{1}=E \cap[a, a+l / 2]$ and $E_{2}=\left[b, q_{2}\right)$. Then $s:=\min _{k=1,2}\left|F^{-1}\left(E_{k}\right)\right| \geq\left|F^{-1}\left(E_{1}\right)\right| / 2$, thus from Lemma 5.4

$$
\begin{aligned}
& \sup _{I^{\prime} \subset I_{2}} \min _{k=1,2} \frac{\left|E_{k} \cap I^{\prime}\right|}{\left|I^{\prime}\right|} \leq \frac{\min _{k=1,2}\left|E_{k}\right|}{l / 2+2 \min _{k=1,2}\left|E_{k}\right|} \leq \frac{2\left|E_{1}\right|}{l} \leq \frac{2|E \cap I|}{l}, \\
& \sup _{I^{\prime} \subset I_{1}} \min _{k=1,2} \frac{\left|F^{-1}\left(E_{k}\right) \cap I^{\prime}\right|}{\left|I^{\prime}\right|} \geq \frac{s}{\left(l^{\prime}-\left|F^{-1}\left(E_{1}\right)\right|\right)+2 s} \geq \frac{\left|F^{-1}\left(E_{1}\right)\right|}{2 l^{\prime}} .
\end{aligned}
$$

And so from the Main Theorem we have

$$
\frac{\left|F^{-1}\left(E_{1}\right)\right|}{l^{\prime}} \leq C\left(\frac{|E \cap I|}{l}\right)^{C /\left\|C_{F}\right\|} .
$$

Since the same estimation holds for $E_{1}^{\prime}=E \cap[a+l / 2, b]$, we obtain (7).

Contrary to the case of homeomorphisms of $\mathbf{R}$, no homeomorphism $F$ : $\mathbf{R} \rightarrow(0, \infty)$ is a BMO map even if $F^{-1}$ is a BMO map. Moreover, there exists a homeomorphism $F:(0, \infty) \rightarrow(0, \infty)$ such that $F$ is a BMO map and $F^{-1}$ is not a BMO map. One such example is given by $F(x)=\log (1+$ $x)$ (cf. Corollary 5.9). This example also shows that we can not drop the condition $2 F^{-1}(I) \subset I_{1}$ in the statement above: Assume that the estimation (7) holds for $I=[\log 2, \log (a+1)]$ and $E=[\log (a / 2+1), \log (a+1)], a>1$. Then

$$
\frac{a / 2}{a-1} \leq K_{0}\left(\frac{\log ((a+1) /(a / 2+1))}{\log ((a+1) / 2)}\right)^{\alpha_{0}}, \quad a>1,
$$

which is a contradiction. Another such example is given by $F:(0, \infty) \rightarrow$ $(0, \infty), F(x)=1 / x$.

Applying (7) to subintervals $I=[a, b]$ and $E=[x, y]$ of $I_{2}, a \leq x \leq y \leq b$, we obtain:

Corollary 5.5. Let $F: I_{1} \rightarrow I_{2}$ be a homeomorphism between open intervals. Assume that $F$ is a BMO map. Then $F^{-1}$ is locally a Hölder continuous function of order $C /\left\|C_{F}\right\|$, where $C>0$ is a universal constant. 
The homeomorphism $F(x)=|x|^{p} \operatorname{sgn} x, p \geq 1$, of $\mathbf{R}$ shows that the estimation above is best possible. (See Example 5.8 below.)

Recall that for a homeomorphisms $G: J_{1} \rightarrow J_{2}$ between (finite) closed intervals, $G$ is absolutely continuous and $G^{\prime}$ is an $A_{\infty}$ weight on $J_{1}$ if and only if $G^{-1}$ is absolutely continuous and $\left(G^{-1}\right)^{\prime}$ is an $A_{\infty}$ weight on $J_{2}$ (cf. Coifman-Fefferman [2]). Thus, under the assumption of the corollary above, $F^{\prime}$ (or $-F^{\prime}$ ) satisfies the $A_{\infty}$ condition uniformly on each $I$ satisfying $2 I \subset I_{1}$. In particular, $F^{\prime} \in L_{\mathrm{loc}}^{p}\left(I_{1}\right)$ holds for some $p>1$, and so $F$ is also locally Hölder continuous. We do not know, however, whether the similar estimation holds or not for the order of $F^{\prime}$. Moreover, from Proposition 5.2 we have:

Corollary 5.6. Let $F: I_{1} \rightarrow I_{2}$ be a homeomorphism between open intervals. Assume that $F$ is a BMO map. Then we have $\left\|\log \left|F^{\prime}\right|\right\|_{*} \leq C$, where $C=C\left(\left\|C_{F}\right\|\right)>0$.

It is easy to see that the corresponding result does not hold for $\log \left|\left(F^{-1}\right)^{\prime}\right|$.

In the rest of the present section, we give a remark on the global behavior of BMO maps. For an open interval $I=(a, b)(\neq \mathbf{R})$, the hyperbolic metric $d s_{h}$ is defined by

$$
d s_{h}(x)=\frac{(b-a) d x}{(b-x)(x-a)} .
$$

The hyperbolic metric is invariant under Möbius transformations of $\overline{\mathbf{R}}$ and comparable with the quasihyperbolic metric $d x / d(x, \partial I)$. Let $d_{h}(x, y)$ denote the hyperbolic distance between $x$ and $y$.

Lemma 5.7. Let $F: I_{1} \rightarrow I_{2}\left(I_{1}, I_{2} \neq \mathbf{R}\right)$ be a homeomorphism between open intervals. Assume that $F$ is a $\mathrm{BMO}$ map. Then

$$
d_{h}(F(x), F(y)) \leq C\left\|C_{F}\right\|\left(d_{h}(x, y)+1\right), \quad x, y \in I_{1},
$$

where $C>0$ is a universal constant.

Proof. Since both the quasihyperbolic metric and BMO are invariant under Möbius transformations, we may assume that $I_{1}=I_{2}=(0, \infty), F$ is sense preserving, $x=1=F(1)$, and $y=a>1$. Applying the Main Theorem with $E_{1}=(0,1]$ and $E_{2}=[F(a), \infty)$, and utilizing Lemma 5.4, we get

$$
\frac{1}{a+1} \leq C\left(\frac{1}{F(a)+1}\right)^{C /\left\|C_{F}\right\|},
$$

hence $F(a) \leq C a^{C\left\|C_{F}\right\|}$, which implies the assertion.

Each hyperbolically Lipschitz continuous function is a BMO function and $F$ preserves the space of all hyperbolically Lipschitz continuous functions if and only if $F$ is hyperbolically Lipschitz continuous. The lemma above shows that the similar result holds for BMO. Note that quasiconformal maps 
satisfy the corresponding estimation with respect to the quasihyperbolic metric. The following example shows that the estimation (8) is best possible.

Example 5.8. Let $F:(0, \infty) \rightarrow(0, \infty), F(x)=x^{p}, p \geq 1 . \quad F$ is the hyperbolic dilation centered at the point 1: $d_{h}(F(x), F(y))=p d_{h}(x, y)$, $x, y \in(0, \infty)$. A simple calculation shows $F$ satisfies (7) with $K_{0}=1$ and $\alpha_{0}=1 / p$. On the other hand, if we set $f(x)=\log x$, then $C_{F}(f)=p f$. Hence, $\left\|C_{F}\right\|$ and $p$ are comparable with universal constant factors. Note that as to the antisymmetric extension $F_{1}: \mathbf{R} \rightarrow \mathbf{R}, F_{1}(x)=|x|^{p} \operatorname{sgn} x$, of $F,\left\|C_{F_{1}}\right\|$ and $p$ are comparable with universal constant factors similarly.

Corollary 5.9. Let $F: I_{1} \rightarrow I_{2}, I_{1}, I_{2} \neq \mathbf{R}$, be a homeomorphism between open intervals. Assume that $F$ is a BMO map. Then $C_{F}$ is a bijection between $\mathrm{BMO}\left(I_{2}\right)$ and $\mathrm{BMO}\left(I_{1}\right)$ if and only if we can take a constant $C>0$ so that for each interval $I \subset I_{1}$ satisfying $d\left(I, \partial I_{1}\right)=|I|$, we have $d\left(F(I), \partial I_{2}\right) \leq C\left|F\left(I_{2}\right)\right|$.

Note that we can take such a constant $C>0$ if and only if

$$
C^{-1} \leq \frac{d_{h}(F(x), F(y))+1}{d_{h}(x, y)+1} \leq C, \quad x, y \in I_{1},
$$

holds for some $C \geq 1$.

Proof. Assume that we can take such a constant $C>0$. Let $f \in \operatorname{BMO}\left(I_{1}\right)$. Then for each $I \subset I_{1}$ satisfying $d\left(I, \partial I_{1}\right)=|I|,\left\|f \circ F^{-1}\right\|_{*, F(I)} \leq C$ holds, thus from Proposition 5.2 we have $f \circ F^{-1} \in \operatorname{BMO}\left(I_{2}\right)$, and so $F^{-1}$ is a BMO map. The converse assertion easily follows from Lemma 5.7.

\section{References}

[1] K. Astala, Remarks on quasiconformal mappings and BMO-functions, Michigan Math. J., 30 (1983), 209-212, MR 85h:30022.

[2] R. Coifman and C. Fefferman, Weighted norm inequalities for maximal functions and singular integrals, Studia Math., 51 (1974), 241-250, MR 50 \#10670, Zbl 291.44007.

[3] M.A. Fominykh, Admissible changes of variables in the class of BMO functions, Math. Notes., 43 (1988), 366-371, MR 89k:42021, Zbl 726.42014.

[4] J.B. Garnett and P.W. Jones, The distance in BMO to $L^{\infty}$, Ann. of Math., 108 (1978), 373-393, MR 80h:46037, Zbl 383.26010.

[5] Y. Gotoh, On the composition of functions of bounded mean oscillation with multivalent analytic functions, J. Math. Kyoto Univ., 29 (1989), 309-315, MR 92c:30028, Zbl 701.30033.

[6] - On the composition of functions of bounded mean oscillation with meromorphic functions, J. Math. Kyoto Univ., 31 (1991), 635-642, MR 93a:30040, Zbl 754.30028.

$[7]$ , BMO extension theorem for relative uniform domains, J. Math. Kyoto Univ., 33 (1993), 171-193, MR 94d:26016, Zbl 783.42013. 
[8] _. On holomorphic maps between Riemann surfaces which preserve BMO, J. Math. Kyoto Univ., 35 (1995), 299-324, MR 96m:30051, Zbl 857.30031.

[9] J. John and L. Nirenberg, On functions of bounded mean oscillation, Comm. Pure Appl. Math., 14 (1961), 415-426, MR 24 \#A1348, Zbl 102.04302.

[10] P.W. Jones, Extension theorems for BMO, Indiana Univ. Math. J., 29 (1980), 41-66, MR 81b:42047, Zbl 432.42017.

[11] _ Homeomorphisms of the line which preserve BMO, Ark. Mat., 21 (1983), 229-231, MR 86a:42028, Zbl 527.42007.

[12] V. Mayer and M. Zinsmeister, Groupes d'homéomorphismes de la droite et du cercle laissant invariant l'espace BMO, Bull. London Math. Soc., 28 (1996), 24-32, MR 96j:30035, Zbl 840.30007.

[13] H.M. Reimann, Functions of bounded mean oscillation and quasiconformal mappings, Comm. Math. Helv., 49 (1974), 260-276, MR 50 \#13513, Zbl 289.30027.

[14] H.M. Reimann and T. Rychener, Funktionen beschränkter mittelerer Oszillation, Lecture Notes in Math., 487, Springer, 1975, MR 58 \#23564, Zbl 324.46030.

[15] W. Smith, Compactness of composition operators on BMOA, Proc. Amer. Math. Soc., 127 (1999), 2715-2725, MR 99m:47040, Zbl 921.47025.

[16] J.-O. Strömberg, Bounded mean oscillation with Orlicz norms and duality of Hardy spaces, Indiana Univ. Math. J., 28 (1979), 511-544, MR 81f:42021, Zbl 429.46016.

[17] A. Uchiyama, The construction of certain BMO functions and the corona problem, Pacific J. Math., 99 (1982), 183-204, MR 84d:42022, Zbl 498.42009.

Received December 21, 1999 and revised June 3, 2000.

Department of Mathematics

National Defense Academy

HASHIRIMIZU 1-10-25

YOKOSUKA 239

JAPAN

E-mail address: gotoh@cc.nda.ac.jp 